\title{
Single Photon Emission Computed Tomography-Computed Tomography (SPECT-CT) Use in Osteosarcoma with Lung Uptake
}

\author{
Khadijah Abdul Hamid', Sazilah Ahmad Sarji \\ ${ }^{1}$ The Department of Nuclear Medicine, Advanced Medical and Dental Institute (AMDI), Universiti Sains \\ Malaysia (USM), Pulau Pinang, Malaysia \\ ${ }^{2}$ The Department of Biomedical Imaging, Universiti Malaya Medical Centre, Kuala Lumpur, Malaysia \\ Email: khadijah ah@amdi.usm.edu.my
}

Received 8 June 2014; revised 7 July 2014; accepted 6 August 2014

Copyright @ 2014 by authors and Scientific Research Publishing Inc.

This work is licensed under the Creative Commons Attribution International License (CC BY). http://creativecommons.org/licenses/by/4.0/

(c) (i) Open Access

\begin{abstract}
A 15-year-old patient with osteosarcoma of left distal femur underwent a bone scan with Tc-99m hydroxymethylenediphosphonate (HDP). Whole body bone scan revealed extensive bone and thoracic metastases. Single Photon Emission Computed Tomography-Computed Tomography (SPECT-CT) of the thorax localized the uptake at pleura and lung nodules. In this case study we want to share our experience using SPECT-CT.
\end{abstract}

Keywords

Tc-99m HDP, Osteosarcoma, Lung Metastasis, SPECT-CT

\section{Introduction}

Osteosarcoma is a primary malignant bone tumour arising from primitive mesenchymal stem cell. This cell is capable of differentiating toward bone, fibrous tissue or cartilage [1]. The most common pathologic variants of osteosarcoma are osteoblastic, chondroblastic and fibroblastic although numerous histologic subtypes exist. Epidemiologically, it is the $6^{\text {th }}$ most common group of malignant tumour in children [1].

Distal femoral and proximal tibial metaphyses is frequently affected as these areas are the region with the greatest growth rate. Usually the diagnosis is first made by a plain radiograph that reveals an ossesous lesion extending into the soft tissue with destruction of cancellous and cortical bone. Magnetic resonance imaging (MRI)

How to cite this paper: Hamid, K.A. and Sarji, S.A. (2014) Single Photon Emission Computed Tomography-Computed Tomography (SPECT-CT) Use in Osteosarcoma with Lung Uptake. Case Reports in Clinical Medicine, 3, 491-495.

http://dx.doi.org/10.4236/crcm.2014.38107 
is then used to assess the extent of the local disease. A computed tomography (CT) scan of the chest is done to identify possible pulmonary metastases and a radionuclide bone scan is arranged to identify bone and soft tissue metastases.

SPECT-CT is effective in differentiating soft tissue lesions such as granulomas from osseous metastases. It can also localize and differentiate between the physiologically active and non-active calcified pulmonary and pleural metastases, as presented in this case report.

\section{Case Report}

A 15 years old boy, referred from a hospital in northern region of Malaysia presented with left hip pain associated with a progressive swelling at the distal part of his left thigh for one week. He was diagnosed with osteosarcoma of left thigh. Initial CT scan of the thorax showed no lung metastasis. He had received 9 weeks of chemotherapy using osteosarcoma protocol (HDMTX) and had radical resection of left femur. Total femur replacement was done after the chemotherapy. Three month after, he complained of shortness of breath, loss of weight and loss of appetite. Chest X ray showed right pleural effusion with several lung nodules (Figure 1). The CT scan revealed metastases to right proximal femur, sternum, T5 vertebrae, left sacral alar and lung. Bone scan using Tc-99m HDP was done to look at whole body bony involvement and it showed extensive bone metastases (Figure 2). In addition to bone metastases, intense radiotracer uptake is seen in the thoracic cavity; hence a SPECT-CT of thorax was done to localize the uptake in the lung (Figure 3 and Figure 4).

\section{Discussion}

Osteosarcoma is a primary malignant tumour of bone originating from primitive mesenchymal stem cell capable of differentiating towards bone. The most common pathologic variants of osteosarcoma are osteoblastic, chondroblastic and fibroblastic. It is the third most common malignant tumour in adolescents and young adults, and also the most common bone tumour in children and adolescents (accounts for approximately $35 \%$ of primary sarcomas of bone) [1]. Approximately $60 \%$ of patients are between 10 and 20 years of age and male to female ratio is $1.3-1.6: 1$ [1].

Distal femoral and proximal tibial metaphyses are the region, which is most commonly affected. These are the regions of the greatest growth rate. It is followed by knee, proximal humeral metaphysis and diaphysis and pelvis.

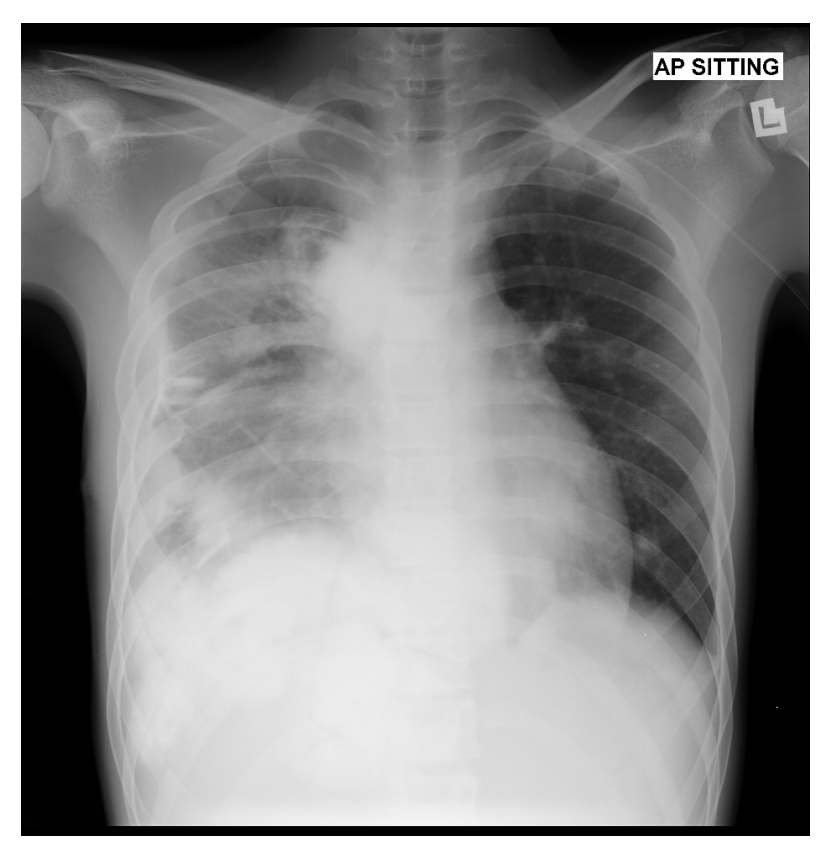

Figure 1. Chest $\mathrm{X}$ ray showed right pleural effusion with underlying right lung collapse. Several nodules of varying sizes are also seen in the left lung. 


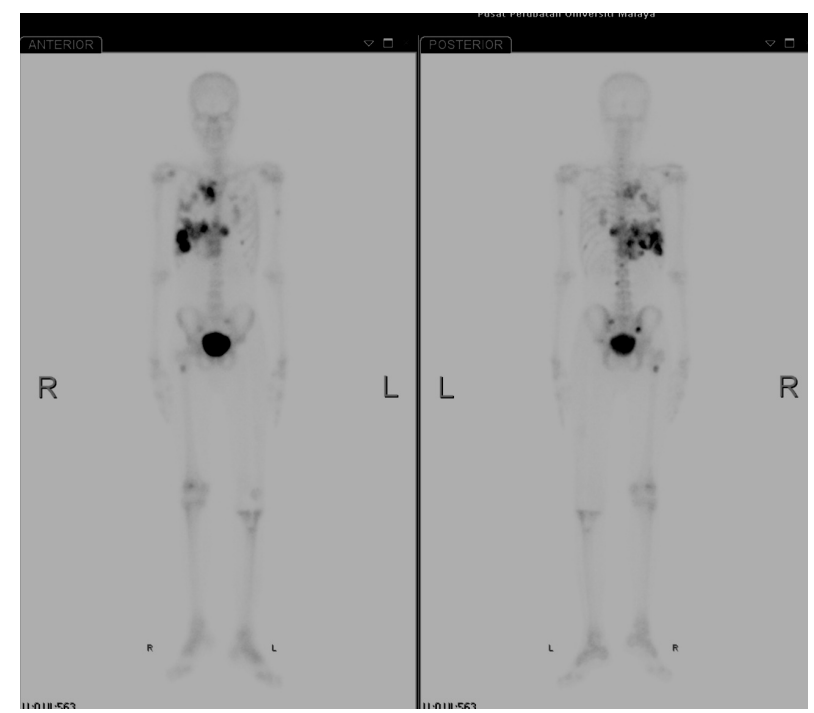

Figure 2. Whole body planar bone scan in anterior and posterior views showed multiple tracer uptake in the right border of scapula, left midshaft of humerus, in the thoracic cavity, multiple bilateral ribs, in the pelvic bones, spine and proximal right femur. There is also a photon deficient area in the left femur in keeping with previous implant.

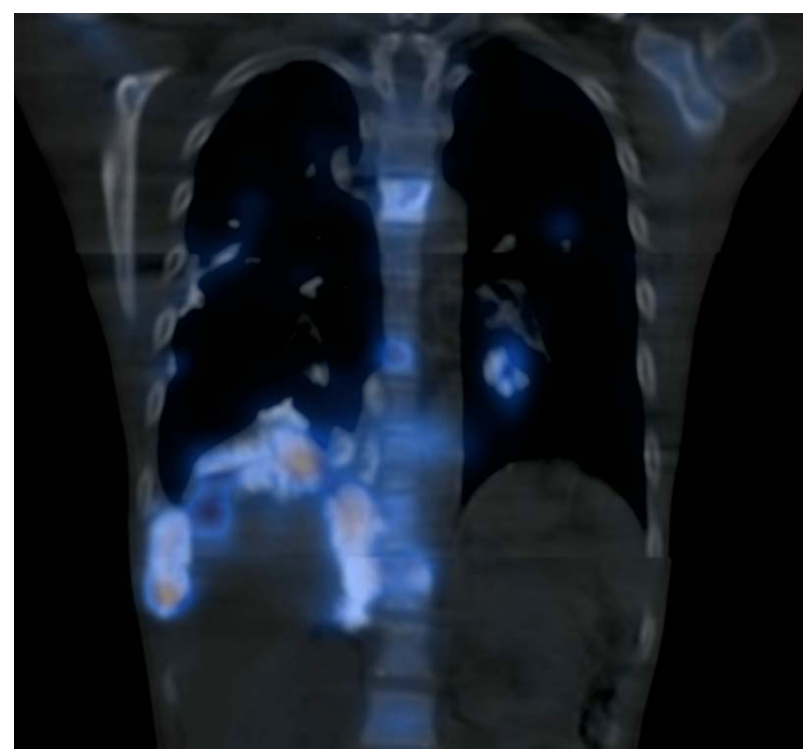

Figure 3. Fused SPECT-CT images of the thorax in coronal view correctly localize the tracer uptake in the bilateral lung nodules, right pleura and the spine.

The most frequent symptom is pain for weeks or months, swelling and loss of function. Loss of weight and loss of appetite are unusual and it indicates metastatic disease. Osteosarcoma frequently metastasis to the lungs followed by bones. Multiple bone metastases also reflect multifocal disease with poor prognosis. It is often diagnosed by history, clinical examination, radiological correlations (CT scan, MRI and bone scan) and tissue biopsy of the affected bone. Radionuclide bone scan is used to scan the whole skeleton, hence to exclude distant bone metastases and recurrence.

The radiopharmaceutical for bone scan is ${ }^{99 \mathrm{~m}}$ Tc-labeled phosphonates (MDP or HDP). Our centre used ${ }^{99 \mathrm{~m}}$ Tc-HDP. The ${ }^{99 \mathrm{~m}}$ Tc-phosphonates accumulate in hydroxyapatite crystal (containing calcium and phosphate ions) 


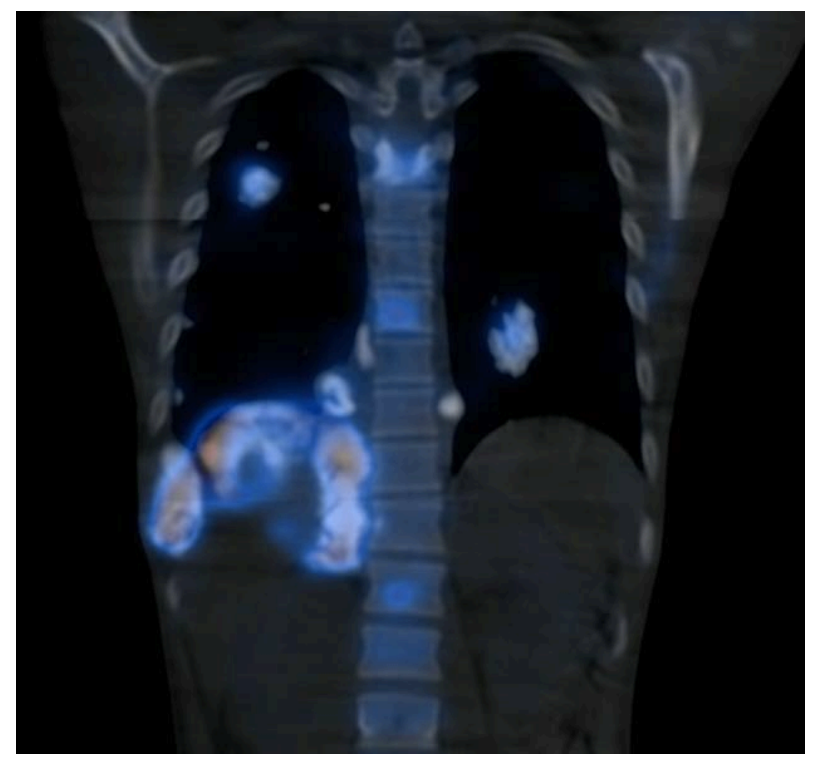

Figure 4. Fused SPECT-CT of thorax in coronal view localized the radiotracer uptake in the right lung nodule (which is much clearer), left lung nodule, right pleura, and the spine.

matrix or in the amorphous (noncrystalline) calcium phosphate. The principle uptake mechanism of the radiotracer is physicochemical adsorption. Metastatic deposits that produce vigorous osteoblastic response will appear as hot spot in bone scan, while the lesions that generate osteolytic reactions may not accumulate the bone radiopharmaceutical [2]. FDG-PET scan is less sensitive than bone scan in detecting bone metastases of osteosarcoma [3].

Introduction of SPECT-CT in the field of medicine in 2004 has made a non-invasive investigation to be able to see both anatomical and functional or metabolic changes of a disease. Both anatomical and functional information is important in the patient management, and most importantly it is done in a single study [4]. Brightview XCT from Philips is used in this case study. CT scan is used for attenuation correction and localization.

In this case study, there are multiple hot spots seen throughout the skeleton in a planar bone scan (Figure 2). From the planar bone scan, we know that the uptake is located in the thorax and other bones, but without SPECT-CT we cannot confirm whether the uptake is actually in the ribs, lung nodules or pleura. The most common causes of accumulation of bone seeking radiopharmaceuticals in extra skeletal tissues include dystrophic and/or metastatic calcification, increased ectopic osteoblastic activity, metastases from bone-forming primary tumors, increase of calcium-binding tissue cations, local $\mathrm{pH}$ changes, inflammation, and increased tumor vascularity [5].

Almost a similar case study was reported before, but it was done without SPECT-CT localization [6]. SPECT-CT has been shown previously to differentiate the uptake in the thorax between the lungs or ribs [7]. This is important in diagnosing whether the increase uptake is in the lung nodule, or due to rib pathology. A fracture rib or a rib metastasis both showed increase in the tracer uptake, but the CT image could differentiate between the two. This is important, as it might change the overall management of a patient. In our case, although the planar bone scan already showed distant bone and lung metastasis, SPECT-CT was done to demonstrate that the lesions in the pleura, lung nodules in bilateral lungs and the spine seen on CT scan were physiologically active osteosarcoma metastases. SPECT-CT is also effective in differentiating soft tissue lesions such as granulomas from osseous metastases, as the former will not show increase in radiotracer uptake.

\section{References}

[1] Kühne, T., Imbach, P. and Arceci, R.J. (2006) Osteosarcoma. In: Pediatric Oncology, Springer, Berlin, Heidelberg, 159-164. http://dx.doi.org/10.1007/3-540-29976-9_14

[2] Elgazzar, A.H. and Shehab, D. (2006) Musculoskeletal System. In: The Pathophysiologic Basis of Nuclear Medicine, Springer, Berlin, Heidelberg, 132-208. http://dx.doi.org/10.1007/978-3-540-47953-6_6 
[3] Franzius, C., Sciuk, J., Daldrup-Link, H.E., Jürgens, H. and Schober, O. (2000) FDG-PET for Detection of Osseous Metastases from Malignant Primary Bone Tumours: Comparison with Bone Scintigraphy. European Journal of Nuclear Medicine and Molecular Imaging, 27, 1305-1311. http://dx.doi.org/10.1007/s002590000301

[4] Beyer, T., Freudenberg, L., Townsend, D. and Czernin, J. (2011) The Future of Hybrid Imaging—Part 1: Hybrid Imaging Technologies and SPECT/CT. Insights into Imaging, 2, 161-169.

[5] Aras, M., Dede, F., Ones, T., Dane, F., Inanir, S., Erdil, T.Y., et al. (2012) 99mTc-MDP-and 18F-FDG-avid Metastatic Liver Lesion: The Similarities and Differences between 2 Modalities. Clinical Nuclear Medicine, 37, 380-381. http://dx.doi.org/10.1097/RLU.0b013e318238f53f

[6] Othman, S. and El-Desouki, M. (2003) Bone Scan Appearance in Aggressive Osteogenic Sarcoma with Pleural, Lung, Bone, and Soft-Tissue Metastases. Clinical Nuclear Medicine, 28, 926. http://dx.doi.org/10.1097/01.rlu.0000093122.94753.37

[7] Mebarki, M., Medjahedi, A., Menemani, A., Betterki, S., Terki, S. and Berber, N. (2013) Osteosarcoma Pulmonary Metastasis Mimicking Abnormal Skeletal Uptake in Bone Scan: Utility of SPECT/CT. Clinical Nuclear Medicine, 38, e392-e394. http://dx.doi.org/10.1097/RLU.0b013e318266cdcb

\section{List of Abbreviations}

SPECT-CT: Single Photon Emission Computed Tomography-Computed Tomography

CT: Computed Tomography

MRI: Magnetic Resonance Imaging

Tc99m: Technetium-99m

MDP: Methylene Diphosphonate

HDP: Hydroxymethylene Diphosphonate

FDG: Fluoro Deoxy Glucose

PET: Photon Emission Tomography

HDMTX: Leucovorin with high dose methotrexate 
Scientific Research Publishing (SCIRP) is one of the largest Open Access journal publishers. It is currently publishing more than 200 open access, online, peer-reviewed journals covering a wide range of academic disciplines. SCIRP serves the worldwide academic communities and contributes to the progress and application of science with its publication.

Other selected journals from SCIRP are listed as below. Submit your manuscript to us via either submit@scirp.org or Online Submission Portal.
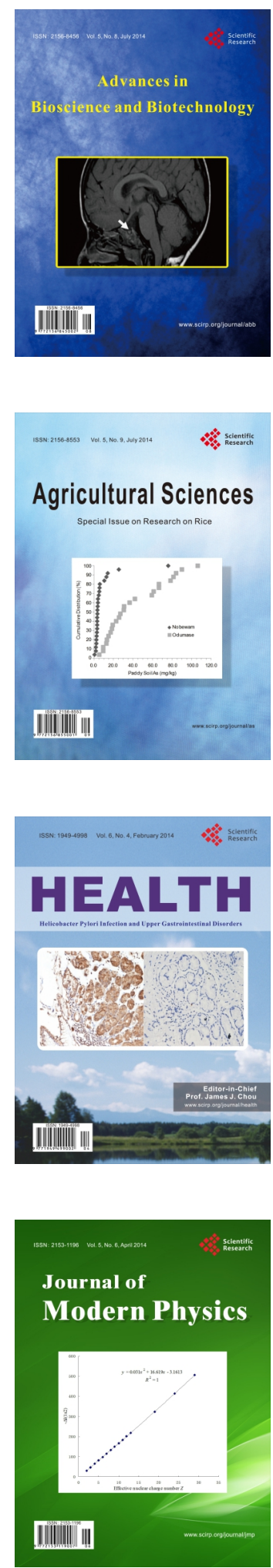
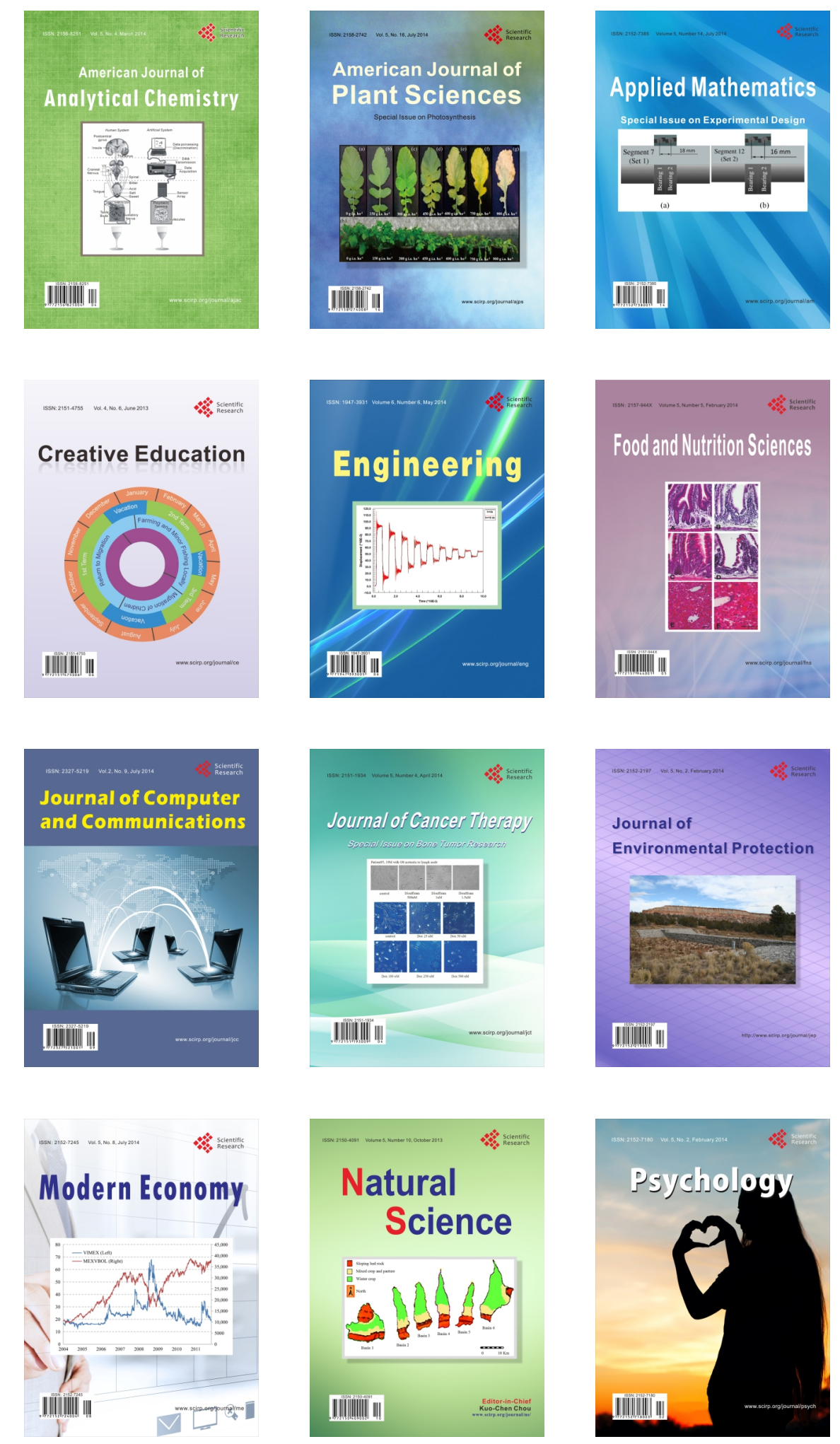